\title{
Electrospun polyvinylidene pyrolidone/gelatin membrane impregnated with silver sulfadiazine as wound dressing for burn treatment
}

\author{
DARIUSH SEMNANI*, NAZANIN POURSHARIFI, NOOSHIN BANITABA and AREF FAKHRALI \\ Department of Textile Engineering, Isfahan University of Technology, Isfahan 845156-83111, Iran \\ *Author for correspondence (d_semnani@cc.iut.ac.ir)
}

MS received 20 July 2017; accepted 3 November 2017; published online 18 May 2018

\begin{abstract}
Nanofibrous membranes used for burn treatment have become widely popular due to their large surface area and high porous structure. In this study, electrospinning was used to fabricate a blended nanofibrous membrane of polyvinylidene pyrolidone (PVP) and gelatin, to use as wound dressing. The physical and mechanical properties of this novel membrane were investigated using SEM, FTIR and tensile tests. Results showed that poor mechanical properties of gelatin, which are preferred in medical applications for curing burns as they allow for antigen activity and skin repair, can be enhanced by adding PVP in the solution. Silver sulfadiazine (AgSD), an antibacterial agent, was also impregnated into the PVP/gelatin nanofibrous structure during electrospinning. The membrane thus fabricated showed antibacterial activities against both the Gram-negative Escherichia coli and Gram-positive Staphylococcus aureus. AgSD release behaviour of fabricated samples indicated short-term drug delivery. It was concluded that the proposed drug-loaded membrane can be used as wound dressing, specifically, in treating skin burns.
\end{abstract}

Keywords. Silver sulfadiazine; nanofibres; gelatin; antibacterial activity; electrospinning.

\section{Introduction}

Nowadays, nanotechnology has found wide applications, specifically in the medical field. Therapeutic, diagnostic and anti-microbial techniques as well as cell repair and drugdelivery systems are some of the more important applications of nanotechnology in medicine [1-3].

Nanomaterials have widespread applications in burn treatment [4]. Burns, as a prevalent injurious incident, may be caused by such varied agents, such as hot liquids, fire flames, chemical materials and radioactive waves [5]. Burn depth depends on the temperature of the inflammable material, skin thickness, exposure duration and skin-heat transfer rate $[6,7]$. Burns are divided into three categories according to the burn depth: epidermal, partial thickness and full thickness. In epidermal burns, the skin colour turns to red and the epidermal layer is injured as well. In the second type, both epidermal and dermal layers are damaged, so that, in addition to changes in skin colour, scorches appear on the skin surface. In full thickness burns, all the layers are injured, so that, the muscles, bones and tendons are marred depending on the burn form and duration $[8,9]$.

Antibiotic ointments and wound dressings are the most well-known means of burn treatment [10,11]. These therapies should continue until the dead tissue falls off and the wound is completely healed. An ideal antibiotic used in such situations should be non-toxic, simple to use, permeable to wound layers and capable of accelerating the wound-healing process. Silver nitrate, mafenide acetate, gentamicin, silver sulfadiazine (AgSD) and betadine are reputable antibiotics [12-15].

In wound dressings, it is of utmost importance to use bandages with good mechanical properties, high permeability, ability to absorb secreted materials and mechanisms to keep the wound area moisturized [16-18]. Recently, nanofibrous structures used as wound dressings and drug-delivery systems have attracted a lot of attention in the medical field due to their high aspect ratio, porous structure and ease of fabrication in different desirable forms $[19,20]$.

Electrospinning of polymeric solutions impregnated with drugs is a convenient way of taking advantage of the beneficial properties of both the embedded drugs and the porous structure of nanofibrous wound dressings. Wei Shao et al [21] fabricated a cellulose/sodium alginate-based composite containing $\mathrm{AgSD}$ for wound-healing applications. Joshua Boating et al [22] used a gelatin/alginate-based composite loaded with $\mathrm{AgSD}$, which showed good capability for infectious sore treatment.

Gelatin has been known for its great role in skin repair due to its superb antigen and antihaemorrhagic activities in addition to its biocompatibility, biodegradability and hydrophilicity [23-25]. However, its application is restricted by its poor mechanical properties and low thermal stability $[26,27]$. 
In this study, electrospinning of polyvinylidene pyrolidone (PVP)/gelatin is used to fabricate a nanofibrous extracellular wound dressing with appropriate mechanical properties and desired degradation rates. Moreover, AgSD is loaded in the nanofibrous membrane, thus produced to take advantage of the beneficial properties of AgSD as an effective antibiotic. All the samples are subsequently characterized by scanning electron microscopy (SEM) and Fourier transform infrared spectra (FTIR). The mechanical properties are also studied to choose the optimum structure based on higher elongation at break and superior tensile strengths. The antibacterial activities of the drug-loaded membrane are examined using the Gram-negative Escherichia coli (E. coli, ATCC 35218) and the Gram-positive Staphylococcus aureus (S. aureus, ATCC 29213). Drug-delivery profiles of AgSD-loaded samples were also studied in phosphate buffer solution.

\section{Experimental}

\subsection{Materials and solution preparation}

PVP and gelatin with average molecular weights of 360,000 and 50,000 Dalton, respectively, were purchased from Sigma Aldrich. Acetic acid was purchased from Merck and AgSD was obtained from Emad Darman Pars Co. Solutions of $\mathrm{PVP} /$ gelatin $9 \mathrm{v} / \mathrm{wt} \%$ were prepared with the five different PVP/gelatin ratios of 100/0, 90/10, 80/20, 70/30 and 60/40. Briefly, a solution of PVP/acetic acid was prepared at room temperature under constant mixing for $\sim 12 \mathrm{~h}$ before gelatin was added. After $24 \mathrm{~h}$ of stirring, a homogeneous solution was obtained for electrospinning.

To fabricate the drug-loaded nanofibrous membranes, the ideal structure of the nanofibrous membrane was identified based on their desired mechanical properties, low fibre diameter and high porosity. This was followed by the addition of AgSD in three different percentages $(0.1,0.2$ and 0.3$)$ of the polymer weight to the polymer solution. AgSD was dispersed well in the solution by ultrasonic stirring at $40 \mathrm{kHz}$ for $90 \mathrm{~min}$. The drug-loaded solution was maintained in the dark to avoid colour changes due to AgSD light sensitivity.

\subsection{Fabrication of non-drug-and drug-loaded PVP/gelatin nanofibres}

Predetermined quantities of the polymer solution were extracted into polypropylene syringes capped with stainless needles (gauge 23). Each syringe was then mounted on the electrospinning apparatus. A negatively charged rotational mandrel was used with an air gap of $16 \mathrm{~cm}$ from the needle tip. To obtain the desired nanofibrous wound-dressing structure, the electrospinning process was carried out by changing voltages $(6.5,8.5$ and $10.5 \mathrm{kV})$ and feeding rates $(0.5,0.53$ and $\left.0.58 \mathrm{ml} \mathrm{h}^{-1}\right)$ at three levels. Electrospinning distance and solution concentration were kept constant for all the samples made.
The same procedure as employed in preparing non-drugloaded samples was used to fabricate three samples of drug-loaded nanofibrous structures with various drug percentages. However, the optimal electrospinning conditions (i.e., flow rate, voltage and PVP/gelatin ratio) for these structures were selected upon analysis of the physical and mechanical properties of non-drug-loaded structures.

\subsection{Characterization}

SEM (HITACHI S-4160) operated at an accelerating voltage of $20 \mathrm{kV}$ was used to characterize the surface morphology of the samples. All the samples were sputter-coated with gold before SEM observations. The mean diameters of the nanofibres and the approximate pore sizes of different samples were determined using the image analysis software (ImageJ, National Institutes of Health, Bethesda, MD). FTIR spectra of all the ingredients (polymers, drug and their combinations) were also provided using a spectrophotometer (BOMEMMB100) in the region from 500 to $4000 \mathrm{~cm}^{-1}$.

\subsection{Tensile properties}

The tensile strengths of the fabricated nanofibrous structures were measured using rectangular specimens $(20 \mathrm{~mm}$ length $\times$ $5 \mathrm{~mm}$ width) under a ZWICK 1432 tensile tester with a $20 \mathrm{~N}$ load cell at a cross-head speed of $10 \mathrm{~mm} \mathrm{~min}^{-1}$. Furthermore, the test was repeated 10 times for each sample to minimize errors.

\subsection{Viscosity and electron conductivity measurements}

Solution viscosities and electron conductivities were measured using a viscometer (Brookfield, USA) with a spindle run at two different speeds (1 and $2.5 \mathrm{rpm}$ ) and a conductivity meter (Jenway, Barloword Scientific Ltd., EU), respectively, maintaining constant electrospinning conditions for all the fabricated structures.

\subsection{Antibacterial activity}

The antibacterial activities of the fabricated membranes were tested against E. coli (ATCC 35218) and S. aureus (ATCC 29213) via the disk diffusion method. Various drug-loaded samples, differing only in their drug ratios, and a nondrugloaded structure, used as the control, were cut into circular shapes $10 \mathrm{~mm}$ in diameter and gently placed over the lawns. The plates thus prepared were placed in an incubator at $37^{\circ} \mathrm{C}$ for $24 \mathrm{~h}$. In addition, the diameter of the inhibition zone for each sample was measured to identify the inhibitory action of the membranes.

\subsection{In vitro drug release measurement}

Drug delivery of three fabricated samples with various ratios of drug loaded was investigated. Phosphate buffer saline 


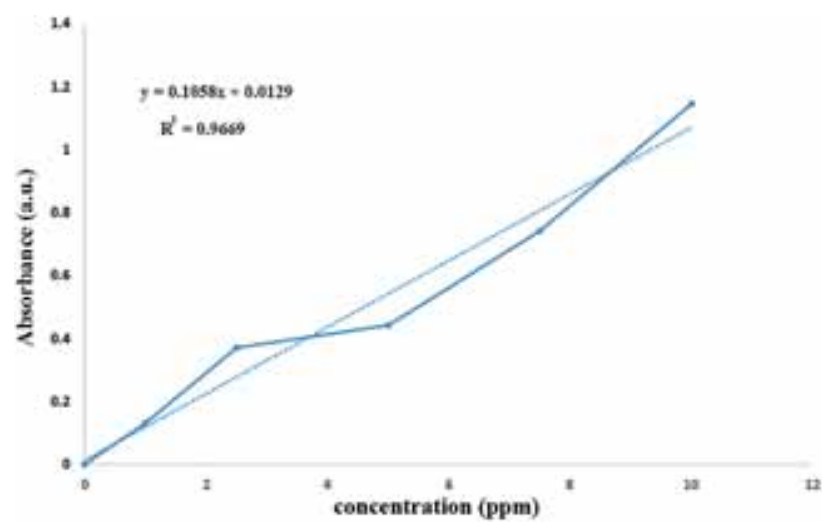

Figure 1. Silver sulfadiazine calibration curve.

solution (PBS, $\mathrm{pH}=7$ ) was applied to simulate drug release behaviour. Circular shape nanofibrous samples with $13 \mathrm{~mm}$ diameter and $1 \mathrm{~mm}$ thickness were placed in dishes containing $10 \mathrm{ml}$ of PBS and incubated with a constant stirring rate at $37^{\circ} \mathrm{C}$. Drug release from electrospun fibres were monitored using ultraviolet-visible spectrophotometer (Shimadzu, UV mini 1240). Beer-Lambert's equation was used to calibration curve determination for several $\mathrm{AgSD} /$ hydrochloric acid concentrations $(1,2.5,5,7.5$ and $10 \mathrm{ppm}$, figure 1$)$. Wavelength of $215 \mathrm{~nm}$ was determined as maximum wavelength of AgSD in hydrochloric acid solvent. Samples were removed from PBS solution at specific times $(35,70,105,140,210,1380,1680$ and $1800 \mathrm{~min}$ ) and examined under ultraviolet-visible (UV) spectrophotometer.

\subsection{Statistical analysis}

Statistical analysis of the data was conducted using the oneway analysis of variance (ANOVA) to determine significant differences among the samples. Duncan and Least-significant difference (LSD) tests were used for comparing the properties of the electrospun samples. In all the analyses, values $<0.05$ were declared as statistically significant.

\section{Results and discussion}

\subsection{Morphology}

Considering the fact that low fibre diameters, high pore sizes and good mechanical properties are significant determinants of the quality of drug-loaded wound dressings, $\mathrm{PVP} /$ gelatin were fabricated in the first step with various ratios $(100 / 0$, 90/10, 80/20, 70/30 and 60/40), and designated by PVP/G-1 to PVP/G-5 (table 1). It is seen that membranes with lower fibre diameters and higher pore sizes were produced with increasing gelatin portions in the solutions. However, the statistical analyses revealed no significant differences between the two groups i.e., PVP/G-2 and PVP/G-3 samples and those of PVP/G-4 and PVP/G-5. PVP/G-4 was chosen as the sample with proper ratios of the blended polymers due to its superior mechanical properties as compared with those of PVP/G-5. Since such electrospinning parameters as feeding rate and applied voltage affect fibre properties like diameter, the PVP/G-4 membrane was also produced under two other applied voltages and two different feeding rates. The electrospun nanofibres obtained were designated as PVP/G-6 to PVP/G-9 (table 1). As expected, PVP/G-8 fabricated at a lower feeding rate and a higher applied voltage was selected as the most desirable structure due to its lower fibre diameter and higher pore size. The SEM images of the electrospun $\mathrm{PVP} /$ gelatin membranes are presented in figure $2 \mathrm{a}-\mathrm{j}$.

\subsection{FTIR}

In the electrospinning process of a solution made by combining two or more polymers, it is vital to make sure that the polymers do not interact or react with each other. The FTIR spectrum was used to identify any likely interactions between PVP and gelatin in the solutions. FTIR spectra of the polymer solutions and drug-loaded polymer solution are presented in figure 3 . The characteristic peaks of PVP, such as $\left(-\mathrm{CH}_{2}\right)$ and $(\mathrm{C}-\mathrm{C})$ are clearly visible at $1425-1475$ and $430-780 \mathrm{~cm}^{-1}$, respectively [28-30]. In addition, gelatin exhibits its distinguished peaks, for instance, of the vibration of carbonyl group $(-\mathrm{C}=\mathrm{O}-)$ at $1648 \mathrm{~cm}^{-1}$ and the bending

Table 1. Average diameters and pore sizes of the fabricated samples.

\begin{tabular}{lccccc}
\hline Code & PVP/gelatin ratio & Voltage $(\mathrm{kV})$ & Feeding rate $\left(\mathrm{ml} \mathrm{h}^{-1}\right)$ & Average diameter $(\mathrm{nm})$ & Average pore size $\left(\mu \mathrm{m}^{2}\right)$ \\
\hline PVP/G-1 & $100 / 0$ & 10.25 & 0.58 & $842 \pm 25.92$ & $1.02 \pm 0.14$ \\
PVP/G-2 & $90 / 10$ & 10.25 & 0.58 & $709 \pm 22.76$ & $2.78 \pm 0.31$ \\
PVP/G-3 & $80 / 20$ & 10.25 & 0.58 & $684 \pm 26.04$ & $2.63 \pm 0.23$ \\
PVP/G-4 & $70 / 30$ & 10.25 & 0.58 & $647 \pm 24.48$ & $2.75 \pm 0.16$ \\
PVP/G-5 & $60 / 40$ & 10.25 & 0.58 & $635 \pm 21.65$ & $2.93 \pm 0.26$ \\
PVP/G-6 & $70 / 30$ & 6.84 & 0.58 & $743 \pm 28.26$ & $1.99 \pm 0.15$ \\
PVP/G-7 & $70 / 30$ & 8.55 & 0.53 & $694 \pm 26.19$ & $2.22 \pm 0.24$ \\
PVP/G-8 & $70 / 30$ & 10.25 & 10.25 & $609 \pm 24.81$ & $3.03 \pm 0.22$ \\
PVP/G-9 & $70 / 30$ & & & $2.91 \pm 0.17$ \\
\hline
\end{tabular}




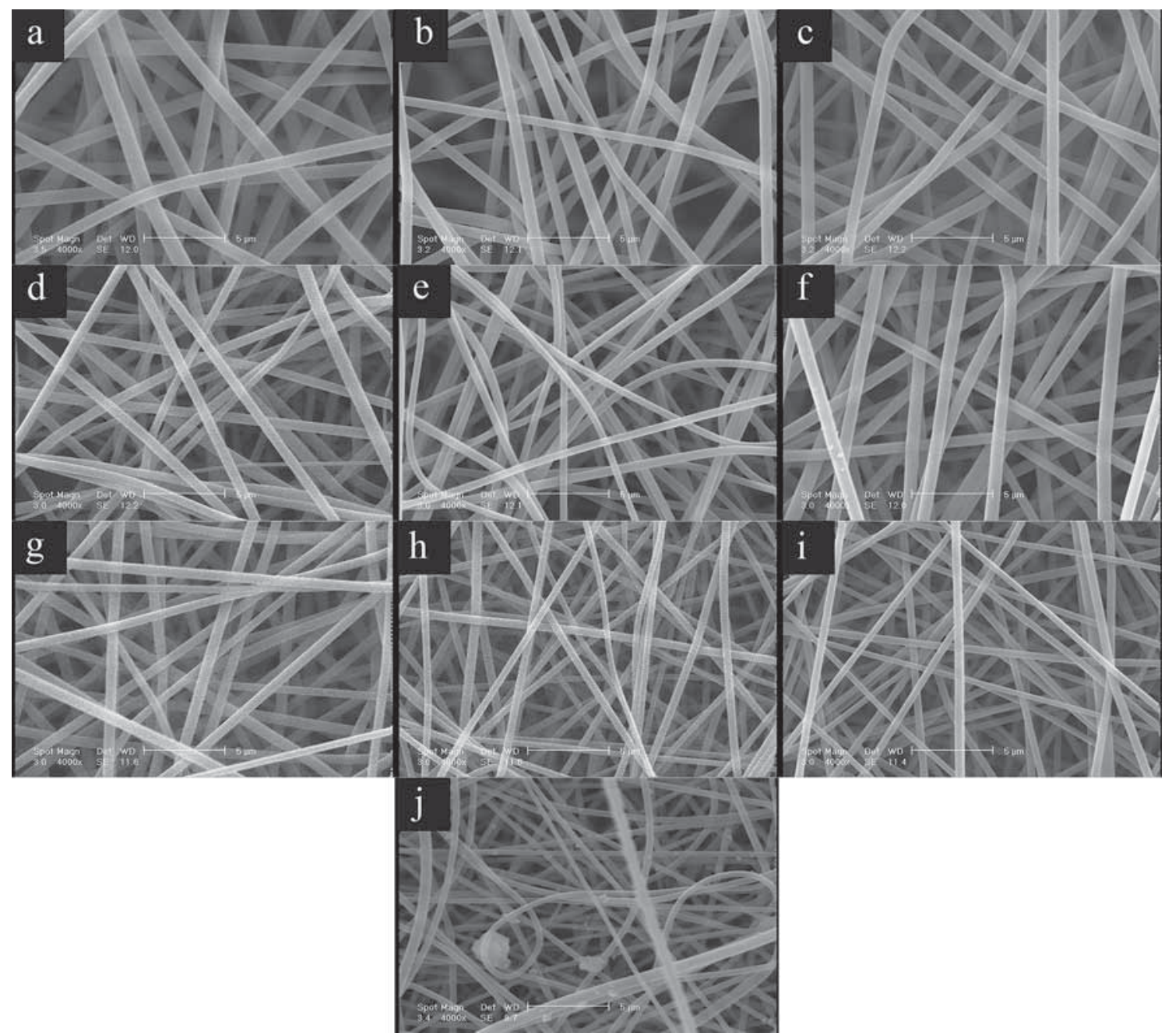

Figure 2. SEM images of PVP/gelatin and AgSD/PVP/gelatin membranes: (a) PVP/G-1, (b) PVP/G-2, (c) PVP/G-3, (d) PVP/G-4, (e) PVP/G-5, (f) PVP/G-6, (g) PVP/G-7, (h) PVP/G-8, (i) PVP/G-9 and (j) AgSD/PVP/gelatin.

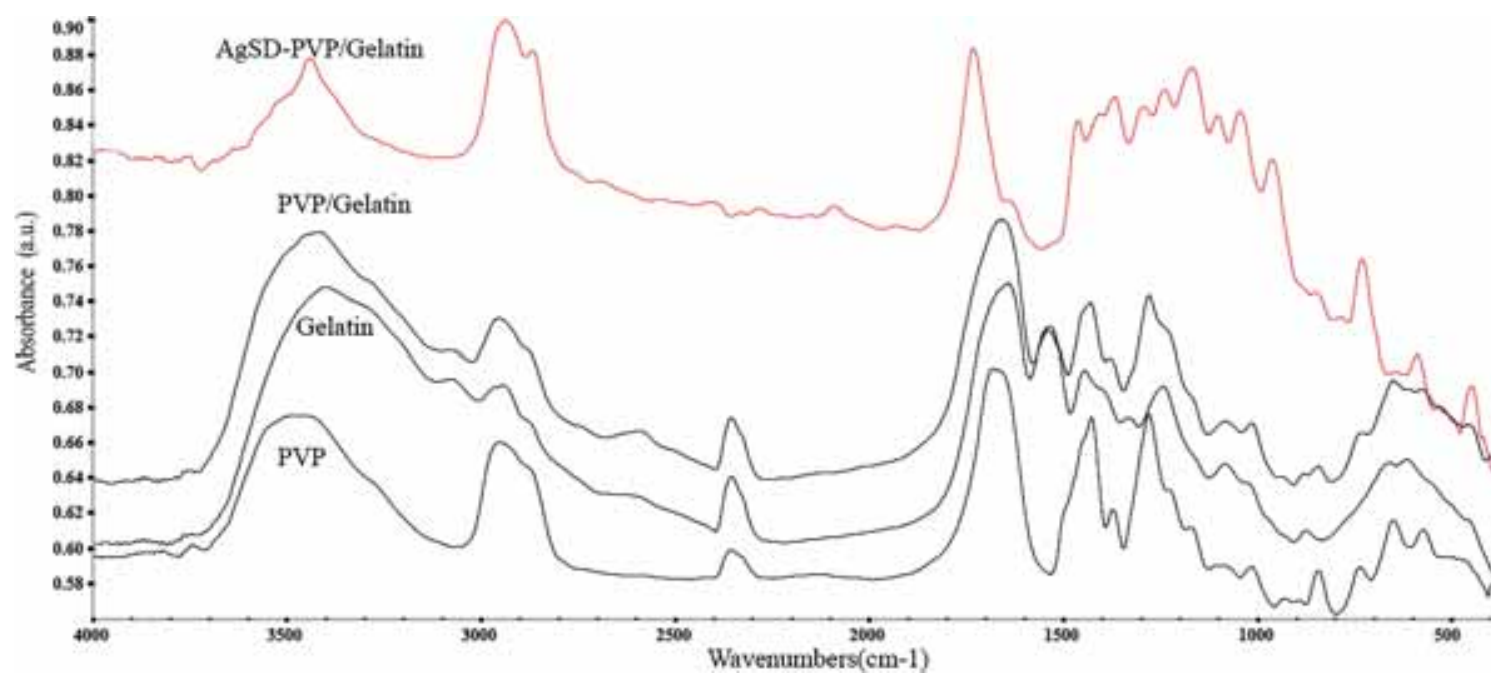

Figure 3. FTIR spectra of (a) PVP, (b) gelatin, (c) PVP/gelatin solution and (d) AgSD/PVP/gelatin. 
Table 2. Solution viscosity.

\begin{tabular}{lcc}
\hline RPM & Non-drug-loaded solutions & Drug-loaded solutions \\
\hline 1 & $619 \mathrm{CP}$ & $445 \mathrm{CP}$ \\
2.5 & $540 \mathrm{CP}$ & $390 \mathrm{CP}$ \\
\hline
\end{tabular}

vibration of (-NH-) at $1540 \mathrm{~cm}^{-1}$ [22]. This is while no FTIR spectra of their combinations are visible in any other specific peak, indicating the lack of interactions between the two polymers in the solution. The characteristic peaks of AgSD drug in the drug-loaded solution, for example, $\left(\mathrm{SO}_{2}\right)$ are visible at $1230 \mathrm{~cm}^{-1}$ and those of the phenyl group at 1552 and $1591 \mathrm{~cm}^{-1}$ [31], confirming the presence of AgSD in the $\mathrm{PVP} /$ gelatin solution and the non-existence of any interactions between them.

\subsection{Viscosity and electron conductivity}

Solution viscosity and its electron conductivity play significant roles in the electrospinning process. Low viscosity and electron conductivity lead to fibre-fabrication problems as they might cause the solution to be sprayed rather than electrospun. Moreover, addition of other substances similar to drugs may lead to undesirable changes in these important properties. Viscosity test results of the PVP/gelatin solutions with and without AgSD are reported in table 2. Clearly, the drugloaded solution exhibits lower viscosity values at different electrospinning speeds. It is also seen that increasing charge density leads to reduced fibre diameter due to the stronger elongation forces exerted on the polymer jet.

The electron conductivity values of the drug-loaded and non-drug-loaded samples were determined to be 14.5 and $13 \mu \mathrm{s}$, respectively. Clearly then, AgSD influences electron conductivity although its effect is negligible. Thus, AgSD affects the viscosity of polymer solutions, which needs to be compensated. One way to maintain constant fabrication conditions is to increase solution concentration. In this experiment, solution concentration was raised to $12 \%(\mathrm{v} / \mathrm{w})$ and the drug-loaded solution was successfully electrospun rather than sprayed.

\subsection{Mechanical properties}

Maximum loads to break and e-modulus of the different nanofibrous membranes, PVP/G-1-PVP/G-9 and $\mathrm{AgSD} / \mathrm{PVP} / \mathrm{G}$ are reported in table 3 . In general, wound dressing employed for burn-treatment applications should be capable of fast degradation, while it does not precipitate. Gelatin is inherently a polymer with poor mechanical properties and sudden degradation. Adding a polymer, such as PVP is an effective method to control the degradation of the nanofibrous structure and the fast release of the
Table 3. Mechanical properties of the samples with different ratios of PVP and gelatin.

\begin{tabular}{lccccc}
\hline & \multicolumn{2}{c}{ F maximum $(\mathrm{cN})$} & & \multicolumn{2}{l}{ Young's modulus (MPa) } \\
\cline { 2 - 3 } Code & Average & CV $(\%)$ & & Average & CV $(\%)$ \\
\hline PVP/G-1 & 44.85 & 20.94 & & 45.64 & 27.12 \\
PVP/G-2 & 35.86 & 18.63 & & 38.19 & 23.93 \\
PVP/G-3 & 26.54 & 17.76 & & 32.53 & 22.81 \\
PVP/G-4 & 23.45 & 19.34 & & 28.96 & 23.48 \\
PVP/G-5 & 17.04 & 21.72 & & 24.44 & 25.63 \\
PVP/G-6 & 24.32 & 24.65 & & 31.07 & 25.96 \\
PVP/G-7 & 21.58 & 20.19 & & 26.84 & 19.37 \\
PVP/G-8 & 23.77 & 23.85 & & 25.69 & 24.12 \\
PVP/G-9 & 24.08 & 19.47 & & 23.57 & 21.78 \\
AgSD/PVP/G & 16.48 & 22.28 & 22.58 & 19.64 \\
\hline
\end{tabular}

drug. As shown in table 3 , increasing the PVP portion of electrospun structures leads directly to improved mechanical properties of the structure, such as higher e-modulus. Our statistical analyses revealed significant differences among all the five membranes with different ratios of the blended polymers (PVP/G-1-PVP/G-5) except for PVP/G-3 and PVP/G-4, which were grouped together by the Duncan's test. However, no significant differences were observed between the PVP/G4 sample and the PVP/G-PVP/G-9 ones, indicating the lack of any effects on mechanical properties due to the changes produced in the electrospinning conditions. As already mentioned above, lower fibre diameters and higher pore sizes in addition to good mechanical properties are important determinant of fibre quality for wound-dressing applications. Based on these vital features, the PVP/G-8 sample was chosen as the best structure for manufacturing the drug-loaded membrane.

Three AgSD-loaded solutions (0.1, 0.2 and $0.3 \mathrm{wt} \%$ ) were fabricated using the fabrication conditions of PVP/G-8. Among these solutions, the PVP/gelatin with $0.1 \mathrm{wt} \%$ of $\mathrm{AgSD}(\mathrm{AgSD} / \mathrm{PVP} / \mathrm{G})$ displayed the proper conditions for electrospinning. The SEM image of AgSD/PVP/gelatin nanofibrous sample is presented in figure $2 \mathrm{j}$. The mechanical properties of this sample were also examined under the same condition as used for the other tested samples. Results revealed no statistically significant differences between PVP/ G-8 and AgSD/PVP/G with respect to their mechanical properties; AgSD may, therefore, be claimed to have no side effects on the mechanical properties of the fabricated membrane.

\subsection{Antibacterial activity}

To study the antibacterial activities of $\mathrm{PVP} /$ gelatin and AgSD/PVP/gelatin membranes, two strains of E. coli (i.e., ATCC 35218) and S. aureus (i.e., ATCC 29213) were selected as Gram-negative and Gram-positive bacteria, respectively, as these are typically associated with infections during 


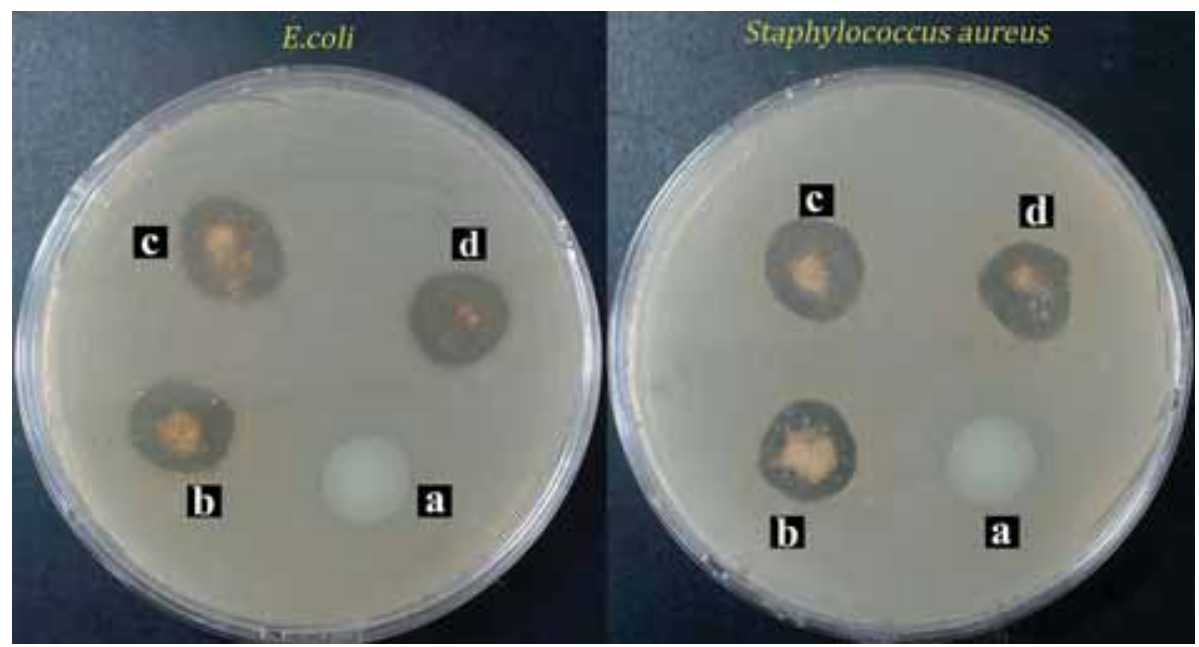

Figure 4. Images of inhibition zones: (a) PVP/gelatin, (b) $0.1 \mathrm{wt} \%$ of AgSD/PVP/gelatin, (c) $0.2 \mathrm{wt} \%$ of AgSD/PVP/gelatin and (d) $0.3 \mathrm{wt} \%$ of AgSD/PVP/gelatin.

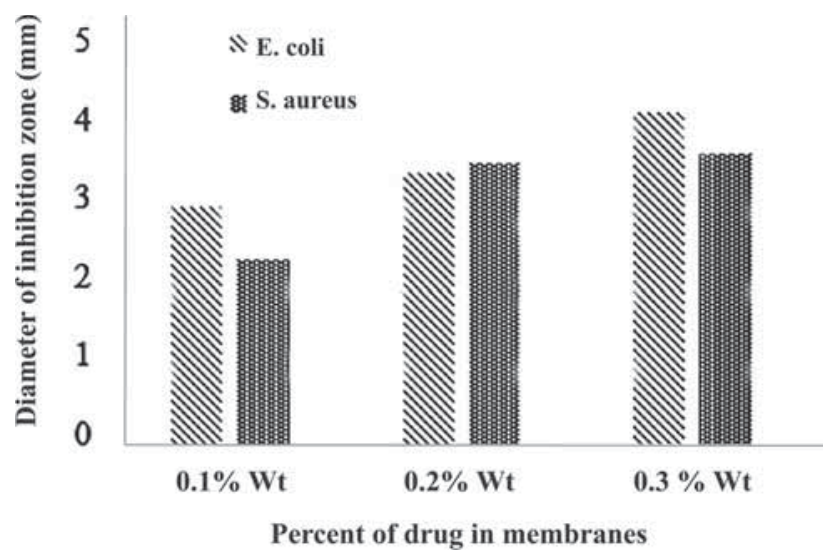

Figure 5. Antibacterial activities of AgSD-loaded membranes with various drug ratios $(0.1,0.2$ and $0.3 \mathrm{wt} \%)$.

wound healing. As already explained above, the antibacterial activities of the samples were measured based on the inhibition zone diameters. Figure 4 shows the antibacterial activity of PVP/gelatin containing different AgSD ratios and the $\mathrm{PVP} /$ gelatin sample used as the control. As expected, the $\mathrm{PVP} /$ gelatin sample showed no inhibition zones, indicating its lack of antibacterial activities against either Gramnegative E. coli or Gram-positive S. aureus. The average diameter of the inhibition zone increased (figure 5) with increasing $\mathrm{AgSD}$ ratio due to the increments in the release of silver ions and sulfadiazine. It is worth noting that membrane shrinkage occurred after placing them on the plates due to PVP hydrophilicity in the membranes.

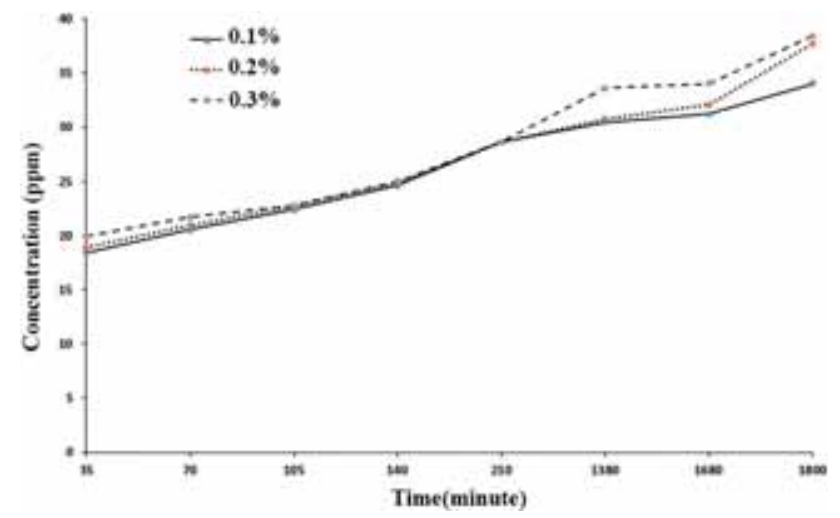

Figure 6. Accumulative release profiles of various AgSD/PVP/gelatin nanofibre mats.

\subsection{Drug release from electrospun PVP/gelatin nanofibres}

Sample PVP/G-8 with three different drug loaded ratios (0.1, 0.2 and $0.3 \%$ ) was selected to study drug-delivery behaviour. The AgSD release profile and calibration curve are displayed in figure 6. Comparison between different electrospun mats showed that sample with more drug-loaded ratio $(0.3 \%)$ has higher drug release through the whole period and hence, greater antibacterial activity, which is along with antibacterial results (figure 5). As it was expected, all samples were degraded after $30 \mathrm{~h}$ due to hydrophilicity of both PVP and gelatin polymers. Notably, AgSD delivery from PVP/gelatin is due to polymer degradation. So, it can be concluded that fabricated wound dressings have the potential for use as temporary drug release, which is highly desirable for burning treatment. 


\section{Conclusion}

Nanofibrous membranes of blended PVP/gelatin were produced and characterized using SEM and FTIR investigations as well as tensile tests. Results confirmed that the presence of PVP was able to improve the mechanical properties of the membranes and to create an appropriate porous structure with suitable degradation rates for wound-dressing applications. The desired electrospun structure was also impregnated with $\mathrm{AgSD}$, which is an effective antibiotic widely used in burn treatment. The nanofibrous membrane showed clear inhibition zones against $E$. coli and $S$. aureus, confirming the AgSD/PVP/gelatin nanomembrane. AgSD release from $\mathrm{PVP} / g$ elatin nanofibrous mat also showed relatively fast drug delivery due to high hydrophilicity of polymers. Antibacterial activity and temporary drug release of AgSD-loaded nanofibrous mats are significant keys to apply as a potentially useful wound dressing, specifically for curing burns.

\section{Acknowledgements}

We would like to acknowledge Emad Darman Drug Company for providing silver sufadiazine during the research, which is free of charge. Also, we pay special thanks to Research Institute for Biotechnology and Bioengineering of Isfahan University of Technology for antimicrobial tests.

\section{References}

[1] Pillay V, Dott C, Choonara Y E, Tyagi C, Tomar L, Kumar P et al 2013 Nanomaterials 20131

[2] Ma Z, He W, Yong T and Ramakrishna 2005 Tissue Eng. 11 1149

[3] Kong H and Jang J 2008 Am. Chem. Soc. 242051

[4] Zhu, L, Liu, X, Du, L and Jin Y 2016 Biomed. Pharmacol. 83 33

[5] Jorge S A and Dantas S R P E 2003 Abordagem multiprofissional do tratamento de feridas (Sao Paulo: Atheneu)

[6] Cho Y S, Choi Y H, Yoon C and You J S 2014 Burns 411
[7] Latarjet J 1995 Burns 21221

[8] Pereira T, Dos Santos D, Lima-Ribeiro M H M, de PontesFilho N T, Carneiro-Leão A M D A and Correia M T D S 2012 BioMed. Res. Inter. 20121

[9] Rangel M F and Pereira A P J T 2007 Bras. Med. 9220

[10] Kumar P M and Ghosh A 2017 Eur. J. Pharm. Sci. 96243

[11] Cribbs R K, Luqutte M H and Besner G E 1998 Surg. Res. 80 69

[12] Nam G, Rangasamy S, Purushothaman B and Song J M 2015 Nanomater. Nanotech. 51

[13] Cherylann M, Gail Land Joel E 2004 Cont. Educ. Top. 74

[14] Mayhall C G 2003 Clin. Infect. Dis. 37543

[15] Benjamin A and Lipsky Christopher H 2009 Clin. Pract. 49 1541

[16] Heo D N, Yang D H, Lee J B, Bae M S, Kim J H, Moon S H et al 2013 Biomed. Nanotech. 9511

[17] May S R 1991 Clin. Mater. 8243

[18] Jayakumar R, Prabaharan M, Kumar P T S, Nair S V and Tamura H 2011 Biotech. Adv. 29322

[19] Valizadeh M, Hosseini Ravandi S A and Ramakrishna S 2013 Tex. Polym. 170

[20] Baker B M, Handorf A M, Ionescu L C, Li W J and Mauck R L 2009 Expert Rev. Med. Dev. 6515

[21] Shao W, Liu H, Liu X, Wang Sh, Wu J and Zhang R 2015 Carbohydr. Polym. 132351

[22] Boateng J, Burgos-Amador R, Okeke O and Pawar H 2015 Biol. Macromol. 7963

[23] Saarai A, Kasparkova V, Sedlacek A T and Saha P 2013 Mech. Behav. Biomed. Mater. 18152

[24] Zhan J and Lan P 2012 Res. Upd. Polym. Sci. 159

[25] Mobarake L G, Prabhakaran M P, Morshed M, Esfahani M H N and Ramakrishna S 2008 Biomaterials 294532

[26] Kuijpers A J, Engbers G H M, Krijgsveld J, Zaat S A J, Dankert J and Feijen J 2000 Biomater. Sci. Polym. Ed. 11225

[27] Ratanavaraporn J, Rangkupan R, Jeeratawatchai H, Kanokpanont S and Damrongsakkul S 2010 Biologic. Macromol. 47 431

[28] Giri N, Natarajan R K, Gunasekaran S and Shreemathi S 2011 Arch. Appl. Sci. Res. 3624

[29] Wang H, Qiao X and Chen J 2005 Mater. Chem. Phys. 94449

[30] Zhang X, Sun N, Wu B and Lu Y 2008 Powder Technol. 182 480

[31] Li P, Wu L and Li B 2016 Mater. Sci. Eng. 6054 